\title{
Global Measles Epidemic Risk: Current Perspectives on the Growing Need for Implementing Digital Communication Strategies
}

This article was published in the following Dove Press journal: Risk Management and Healthcare Policy

\author{
Elena Bozzola' \\ Giulia Spina' \\ Alberto Eugenio Tozzi ${ }^{2}$ \\ Alberto Villani' \\ 'Pediatric Unit, Bambino Gesù Children's \\ Hospital, IRCCS, Rome, Italy; \\ ${ }^{2}$ Multifactorial and Complex Diseases \\ Research Area, Predictive and Preventive \\ Medicine Unit, Bambino Gesù Children's \\ Hospital, IRCCS, Rome, Italy
}

\begin{abstract}
A safe vaccine against measles has been available and globally recommended since 1974. The World Health Organization established measles elimination as a goal for 2020 but, unfortunately, this objective has not been achieved yet and outbreaks still occur. Herd immunity, ie, a population immunity higher than $95 \%$, is required to stop the measles virus transmission. Communication plays a crucial role in immunization strategy to obtain high coverage levels, as it helps to fight barriers against vaccination. Delay and refusal of measles vaccination have become widespread due to misinformation, fake news and barriers to effective communication. This phenomenon has been defined as "vaccine hesitancy" and is considered as one of the top ten risks for global health. The alleged association between measles vaccination and autism has caused a sharp decline in vaccination rates. In this current situation, mass communication integrated into public health policies is fundamental to sway people's positive attitudes toward vaccination. Digital communication strategies based on social media and other internet platforms may represent useful tools to promote immunization and discourage skepticism and complement information provided by health-care professionals who have been considered as the most credible source on risk/benefits on vaccines for families. Digital communication strategies that may help supporting the measles elimination strategy include monitoring information needs online, integrating digital communication into immunization programs, involving a multidisciplinary group in communication, developing content that balances facts with positive messaging, using multiple communication channels. Further research activities should be promoted in the field of effective communication for immunization.
\end{abstract}

Keywords: measles, children, vaccination, health

\section{Introduction}

Measles is an eradicable, highly contagious, vaccine-preventable disease that still causes nearly 100.000 deaths every year, despite the availability of immunization. ${ }^{1}$

The alleged adverse events associated with the available vaccine have hampered the success of the global immunization strategies, undermining the trust on measles immunization, despite the large body of evidence about measles vaccine safety. ${ }^{2}$

Communication plays a crucial role in every immunization strategy. Taking into account the public's emotions and worries is a fundamental element for decisionmakers regarding immunization, and making information about vaccines efficacious and transparent.

False information about vaccine safety affects immunization strategies and can change the perception of the public about vaccine safety leading to vaccine
Correspondence: Elena Bozzola Bambino Gesù Children Hospital, Piazza S Onofrio 4, Rome 00165, Italy Tel +39(0)668592744

Email elena.bozzola@opbg.net
Risk Management and Healthcare Policy 2020:13 2819-2826

2819 
hesitancy, to a decrease of vaccine coverage, and finally to the resurgence of vaccine-preventable diseases. While debunking fake news regarding vaccines seems an obvious counter reaction, studies have shown how people tend to decline information corrections which are distant from their beliefs. ${ }^{3}$

The increased availability of the internet and digital tools has enhanced the interest towards using social media and other digital channels for vaccine communication strategies. To address the challenges of digital communication strategies for immunizations, it is essential to integrate epidemiological information about vaccine-preventable diseases with other information relevant to digital marketing.

As this is an emerging field in vaccinology, in this review we focus on prevention of measles describing the main epidemiological characteristics, the changes in public trust caused by fake news, and the potential effects that digital communication may have to improve and sustain measles immunization.

\section{Measles Epidemiology}

Measles occurs in susceptible populations, even when the proportion of susceptible individuals is lower than $10 \%$, due to its high level of contagiousness. ${ }^{4}$ Measles complications include acute lower respiratory infections, otitis media, neurological complications such as post measles encephalitis, SSPE and measles inclusion body encephalitis, croup, diarrhea, visual disturbances, which may lead to morbidity and mortality. ${ }^{5}$ In order to stop viral circulation, a population immunity of greater than $95 \%$ is needed ${ }^{6}$ Measles eradication is biologically and technically feasible because humans are the only natural hosts for measles virus, only one serotype of the measles virus exists, and measles virus is genetically stable. ${ }^{7}$

The currently available vaccine, an attenuated viral vaccine administered with rubella, mumps or varicella, is safe, effective and inexpensive and has prevented a significant number of deaths and disabilities since its introduction, ${ }^{6}$ with a decreased measles mortality worldwide by $84 \%$ between 2000 and $2016^{8}$

Despite the global effort and the high immunization coverage achieved in most areas of the world, several outbreaks have still occurred in recent years, such as in Venezuela in 2017, Madagascar, Philippines and Brazil in $2019 .^{8-11}$ Sub-optimal immunization programs may also reestablish transmission in countries that have previously eliminated endemic virus transmission, including Mongolia in the Western Pacific region, Albania, Czech Republic, Greece, and the UK in the European region. ${ }^{7}$

More recently, there has been an increasing concern about the effect of COVID 19 on immunization coverage for measles. At the beginning of 2020, a 1\% decline of measles vaccinations was detected in England, which later on raised to $7.2 \%$ after social distancing measures and the closing of schools policies were established. In week 13 of 2020 vaccination rate decrease peaked to $24.2 \%$ decline, in parallel with the increasing epidemic emergency. ${ }^{12}$ It has been suggested that, while recommending social distancing and other preventive measures, communication on the need to continue routine vaccination programs was not explicit. $^{12}$ WHO estimates that by the end of October 2020 delays in vaccination campaigns in 26 countries have led to over 90 million children missing scheduled measles vaccine dose, increasing the risk of measles. ${ }^{13}$

\section{Current Immunization Strategies}

Measles has been included in global programs for immunization since 1974, when the 27th World Health Assembly established the Expanded Program on Immunization to ensure that all children, in all countries, benefited from this life-saving vaccine. ${ }^{6}$

Hence, vaccination for all susceptible children and adults for whom measles vaccination is not contraindicated, is globally recommended. Universal immunization of all children with the administration of 2 doses of the measles vaccine, either alone, or in a measles-rubella, measles-mumps-rubella, or measles-mumps-rubella-varicella combination, should be the standard for all national immunization programs. ${ }^{6}$

Measles has been targeted for elimination by 2020 in all of the World Health Organization regions. ${ }^{6}$ Unfortunately, this objective has not been achieved yet due to insufficient immunization coverage, and insufficient control of emerging clusters. As the original target for measles elimination had to be postponed, new strategies need to be adopted. Resuming progress toward measles elimination will require not only the improvement of immunization programs and disease surveillance but also of communication strategies. Moreover, as the success of global immunization strategies lays on the coordinated efforts in all countries, it is essential that all actions should be standardized.

The objective of mass immunization programs is achieving very high coverage rates to establish herd immunity in the population, ie, decreasing the proportion of susceptible individuals to levels that do not allow measles 
to circulate. ${ }^{14}$ Due to suboptimal immunization coverage, or to non-homogenous coverage in different geographic areas, measles still circulates in many areas of the world and outbreaks may happen even when routine immunization coverage is very high. As with any transmissible disease, when infected individuals enter sufficiently large pockets of susceptible individuals, outbreaks occur. In addition to routine immunization programs, the World Health Organization also recommends enhancing preparedness for measles outbreak response.

Sometimes outbreaks occur in groups that are under immunized for ethnic, religious, philosophic, or economic reasons, which calls for targeted strategies. Moreover, when susceptible individuals have difficulty accessing health services and are of a poor socioeconomic status, they are consequently prone to more severe manifestations of measles and its complications. ${ }^{15}$

Strategies to achieve measles elimination may require a substantial effort involving several integrated actions. The World Health Organization strongly recommends maintaining $\geq 95 \%$ coverage with two doses immunization by an active vaccine to subjects without vaccine or immunity against measles, and who are at increased risk of measles infection and transmission, such as health-care workers, people working in tourism and transportation, and international travelers. ${ }^{6}$

Moreover, epidemiological surveillance for patients presenting with fever and rash along with laboratory diagnosis, are crucial tools for early case identification. This helps to control transmission through the isolation of susceptible contacts since measles cases are potentially infectious from the prodromal phase, 4 days before the onset of rash. ${ }^{16}$

Finally, the identification of susceptible communities with suboptimal immunization coverage is required to interrupt indigenous measles transmission. Analysis of measles case-based surveillance data not only guides strengthening of routine immunization efforts by identifying high-risk groups and areas but can also indicate areas with likely underperforming disease surveillance. ${ }^{17,18}$

The Global Measles and Rubella Strategic Plan 2011-2020 established measles elimination goals by adding supplementary immunization activities, such as mass immunization campaigns to immunize both children and adults missed by routine vaccination or without immune protection. Six guiding principles have been discussed throughout the Plan: country ownership, shared responsibility and partnership, equity, integration, sustainability, and innovation. ${ }^{19}$
In some countries, compulsory measles vaccination programs have been proposed as a strategy to achieve higher immunization rates more quickly and in favor to the right of protection via herd immunity of the individuals who cannot be vaccinated. ${ }^{20}$ Proposed strategies to obtain higher vaccine coverage include increasing the difficulty of obtaining vaccine exemptions. ${ }^{21}$

The current schedule for routine immunization includes the first dose of the measles vaccine at 12 months of age with a second dose administered at $15-18$ months. ${ }^{6}$ The first administration dose can be at 9 months of age for children in countries with endemic measles. It can be given at 6 months or earlier to HIV children in areas with outbreaks as well as to children who are at high risk for contracting the disease such as refugees. Administration of the measles vaccine before 12 months of age may result in lower antibody response. ${ }^{1}$

During pregnancy, immunized women transfer antibodies leading to onset of protection until around 4 months of age. After birth, the transferred antibody titers progressively wane so that most infants have non-protective titer of antibody left by the age of 5 months. A still unsolved problem is represented by the maternal antibodies which confer protection against infections early in life but inhibit vaccination response. So, in developing countries, the need for early induction of protective neutralizing antibodies after vaccination is hampered by maternal antibodies. Actually, no effective immunization strategy for vaccination in the presence of maternal antibodies has jet been defined because the underlying mechanism of interference is not known. ${ }^{22}$

\section{Measles Vaccine Misconceptions}

The reservations regarding the measles vaccination mainly question the vaccination's safety and stress possible longterm adverse effects, while assuming that measles is a harmless childhood disease. ${ }^{23-25}$ The worry on exposing a healthy child to the risk of adverse effects of a vaccination is understandable and is known in the behavioral sciences as omission bias. ${ }^{26}$

There are several examples of omission bias throughout the history of vaccines, but immunization against measles offers probably the most impactful case study. In the late 1990s, a research paper suggesting a link between the measles vaccine and the development of autism was published in The Lancet. Although the study was retracted, its publication was sufficient to trigger the biggest controversy ever involving immunization, which fueled the 
publication of an incredible number of news stories regarding the measles vaccine. ${ }^{27}$ Several subsequent studies demonstrated the absence of any link with autism. Among them, a retrospective study performed in Denmark on 537,303 children born between 1991 and 1998, demonstrated the absence of any association between measlesmumps-rubella (MMR) vaccine and autism. ${ }^{28}$ Moreover, a recent meta-analysis conducted in the US, involving 5 large cohort studies and 5 case-control studies, with a total of 1,266,327 children, documented no association between MMR vaccination and autism. ${ }^{29}$

Such intense discussion in the media and on social networks caused a drop-in vaccination uptake in the UK to levels as low as $79.9 \%$ in $2003-2004,{ }^{30}$ and a sudden increase in measles cases. ${ }^{31}$ The effect of the MMR and autism saga also spread across Europe with long-lasting effects. The MMR and autism controversy can partially explain other outbreaks. In 2017, a large outbreak occurred in Minnesota, mostly involving the Somali American population. ${ }^{32}$ The spread of misinformation about MMR and autism in this community may explain the sharp decline of immunization rates in this population, which previously had high coverage rates. ${ }^{32}$

One of the most recent measles outbreaks was observed in Disneyland in Anaheim, California, where half of the measles cases were in unvaccinated persons, most of whom were eligible for vaccination yet intentionally remained unvaccinated. ${ }^{33}$ The majority of the patients with reported measles in the 2015 outbreaks were either unvaccinated $(45 \%)$ or had unknown vaccination status $(38 \%)^{33}$

This observation triggered a strong discussion in the mainstream media and on social networks about vaccination hesitancy and controversial legislation, ${ }^{34}$ highlighting how media can affect vaccine attitudes. ${ }^{35}$

\section{Online Misinformation on Measles Vaccine}

Low vaccine confidence is associated with misinformation and lack of access to accurate information. Web and social media have become powerful amplifiers of shocking news and play a major role in spreading misinformation and fake news on vaccines. ${ }^{36,37}$ On the other hand, several studies demonstrate that parents trust their child's doctor as an information source about vaccination. ${ }^{34}$ False information online may supplement that from health providers and conflict with them, resulting in confusing parents and culminating in vaccine hesitancy. ${ }^{34}$ Of note, staunch vaccine objectors have a generally high level of education and yet are hard to convince even when presented with scientific evidence. ${ }^{38}$

As internet users leave digital traces behind, the use of web metrics has become increasingly popular for monitoring vaccine confidence on the web. A recent study showed, through hashtag analysis, how negative comments about vaccines on Twitter increased in coincidence with measles outbreaks and the publication of anti-vaccine books and movies. ${ }^{39}$

The study of vaccine information dynamics on the web is interesting as it allows for the possibility of studying how individuals behave when discussing vaccinations online. A recent study performed on Facebook showed that antivaccination users access more information sources than those who support immunizations and are more engaged in the community. ${ }^{40}$ Inevitably, this behavior leads to polarization.

Online misinformation is obviously relevant during outbreaks, when people seek credible information in an emergency. For this reason, timely information from credible sources is crucial. ${ }^{41}$

Psychological research has offered useful concepts on how to focus messages in response to misperceptions suggesting to provide the general public with insights that help them debunking "no-vax" topics and sustain trust in health authorities and the immunization programs. ${ }^{42}$ Scientific research on communication shows that the quality of the evidence not only influences the audience's attitudes towards a health treatment but also increases credibility. ${ }^{43}$

Messages based on scientific approach may have a greater influence on people's attitude toward vaccinations in comparison to affective associations or simple allegations used by deniers. Consequently, underlining scientific messages, based on knowledge and facts, may help improve people's trust on immunization.

\section{Communication for Measles Immunization}

The available scientific knowledge about communication promoting immunizations suggests that decision-making toward immunization depends on multiple factors. Much of the effort in communication activities focuses on providing evidence-based facts or statistics to drive decisions which should be presented to the public. ${ }^{44}$

However, decisions regarding immunization may also be driven by emotions and, consequently, by specific local 
environments and other circumstances. Often health-care providers and public health agencies tend to disregard the emotional part of public perception, missing the possibility to create an effective dialogue with them. ${ }^{44,45}$

Health-care providers play a key role in fighting barriers to vaccination as patients' choices are mostly influenced by their pediatrician's view of the vaccinations.46

A meta-analysis indicated health-care professionals as the most credible source on risk/benefits of vaccines. ${ }^{45}$ In cases of concerns about vaccines or vaccine hesitancy, a positive dialogue-based approach, and multimodal strategies (for example, flyer plus dialogue) have been demonstrated to be most effective. ${ }^{46}$

As fake news is widely disseminated on the web, a strong effort is required in debunking false information through digital means. Evidence exists that echo chambers for negative and positive impressions on vaccines are well established on the web with strong polarization. ${ }^{47}$ Individuals belonging to these echo chambers tend to select and spread information that confirms their own beliefs (confirmation bias) and which reinforce their position. ${ }^{48}$ Debunking in this context may have no effect or may even reinforce the beliefs of some individuals. ${ }^{49}$

There is room for research to understand if marketing strategies with positive messages about vaccines compared to fact checking and debunking, may be more effective in changing behaviors about vaccines. ${ }^{49}$

Much of the focus of communication strategies for vaccines is on vaccine hesitancy. The World Health Organization has established vaccine hesitancy as one of the top ten risks for global health. ${ }^{50,51}$ The president of the American Academy of Pediatrics requested a partnership with Google, Facebook and Pinterest in order to ensure the content shared on these platforms use only science-based information. ${ }^{51}$ In response, Facebook declared that pages with anti-vaccine misinformation would be eliminated. ${ }^{51}$

On the other hand, social media campaigns conducted on the web to promote immunizations seem to have a measurably positive effect. ${ }^{52}$ Communication strategies that resonate on social media may clarify and emphasize unfamiliar information, facilitating its comprehension, and influencing parents' decisions on vaccination. ${ }^{53}$

The WHO has recently conducted a project based on a global network of websites, aimed at providing highquality, standardized information on vaccine safety. ${ }^{54}$ In this project, web analytics were used to tailor vaccine safety information. As a result, the potential to enhance immunization campaigns with the support of coordinated and standardized digital tools delivered by large networks was demonstrated.

The challenge of efficacious communication for measles immunization has been addressed by comprehensive reference documents. ${ }^{55}$

Despite the fact that many principles of communication for vaccine promotion have been clearly established, effective strategies for measles immunization require adaptation, flexibility, and tailoring, which make them difficult to standardize. An essential element to guide immunization strategies is that of listening to the public. Monitoring public confidence on vaccines online through social media listening is of paramount importance to plan the right strategies with the appropriate content. ${ }^{46,55,56}$ It is increasingly evident that these data complement those derived from surveillance systems since an increase in hesitancy may anticipate a drop in vaccine coverage and an increase of incidence of measles. Online discussions on vaccines seem reasonably representative of what happens in the real world, they reflect changes in real time, and social media monitoring is cost effective. Moreover, several technological tools may be used for interpreting social interactions on the web, including natural language processing techniques, which may help in detecting hot topics in the immunization discourse. Finally, a systematic application of search engine optimization techniques helps to maintain information published online at the highest rank.

As vaccine hesitancy may occur in different subpopulations, a well-established partnership is essential to share common strategies and to link with stakeholders that may help to reach out to specific segments of the community with the appropriate digital tools. Partners in communication activities should not be limited to health professionals but should expand to include associations and communities that may be pivotal in transmitting information to ethnic, religious, or other subgroups of the population. Indeed, as in every communication activity, in segmenting the target audience, it is extremely important to use the right information channels and tailored content and adapt them to the background beliefs of the target groups. Online marketing strategies largely use this approach and select online communities for their purposes. For example, Instagram and TikTok are social media platforms mostly used by very young people, with a prevalence of women, while adults mostly use Facebook. Using multiple communication channels, including non-digital ones, is however very important to reach a wider audience, 
including those that may be marginalized because of the digital divide.

Combining fact checking with positive messages about the measles vaccine seems reasonable, while it seems impractical to focus communication activities on the debunking of fake news only. Different formats, from audio-visual to simple or short text, and content that require a short time to understand, may help to reach a larger audience.

Integrating communication activities into surveillance strategies, in addition to the surveillance of vaccine adverse events, is very important to inform comprehensive immunization strategies. Looking simultaneously at the epidemiological features of segments of the population, and at their information needs and behavior, may allow for predicting potential outbreaks and prioritizing and tailoring communication efforts. Digital communication requires a multidisciplinary approach in which epidemiologists, clinical doctors and those involved with public health, work side by side with communicators and marketing experts, data scientists and journalists. An ideal approach would be also including laypersons in the communication-working group to facilitate translation of key messages in the field. However, the most important aspect is producing new evidence about efficacious communication for the measles vaccine and for immunizations in general. Planning experimental studies embedded into immunization campaigns and in other communication processes is strongly needed to refine and inform future strategies. Along this line, it must be underlined that communication requires resources for the integration with measles immunization strategies and for applied research. Finally, digital communication offers tools to timely adapt to special circumstances such as the COVID 19 pandemic, and avoid a decline in immunization rates. If the global community will be successful in providing effective communication for measles immunization and allow the public to understand the key facts of immunization strategies, measles elimination will be easier to achieve.

\section{Conclusions}

Reinforcing digital communication strategies that may help support the measles elimination strategy is urgently required and convenient. The integration of digital communication strategies into immunization programs may require the involvement of a multidisciplinary group and the use of multiple communication channels.

\section{Abbreviation}

MMR, measles-mumps-rubella.

\section{Author Contributions}

All authors made a significant contribution to the work reported, whether that is in the conception, study design, execution, acquisition of data, analysis and interpretation, or in all these areas; took part in drafting, revising or critically reviewing the article; gave final approval of the version to be published; have agreed on the journal to which the article has been submitted; and agree to be accountable for all aspects of the work.

\section{Funding}

There is no funding to report.

\section{Disclosure}

Alberto Eugenio Tozzi reports personal fees from Roche, Novartis, and Astra Zeneca, outside the submitted work. The authors report no other potential conflicts of interest for this work.

\section{References}

1. Moss WJ, Scott S. The Immunological Basis for Immunization Serie. Module 7: Measles-Update 2009. Geneva: World Health Organization; 2009.

2. Wilder-Smith AB, Qureshi K. Resurgence of measles in Europe: a systematic review on parental attitudes and beliefs of measles vaccine. J Epidemiol Glob Health. 2020;10(1):46-58. doi:10.2991/ jegh.k.191117.001

3. Nyhan B, Reifler J. When corrections fail: the persistence of political misperceptions. Polit Behav. 2010;32(2):303-330. doi:10.1007/ s11109-010-9112-2

4. Chen RT, Goldbaum GM, Wassilak SG, Markowitz LE, Orenstein WA. An explosive point-source measles outbreak in a highly vaccinated population. Modes of transmission and risk factors for disease. Am J Epidemiol. 1989;129(1):173-182. doi:10.1093/oxfordjournals.aje.a115106

5. Perry RT, Halsey NA. The clinical significance of measles: a review. J Infect Dis. 2004;189(Suppl 1):4-16.

6. WHO. Measles vaccines: WHO position paper-april 2017. Wkly Epidemiol Rec. 2017;92:205-227.

7. Durrheim DN. Measles eradication-retreating is not an option. Lancet Infect Dis. 2020;20(6):e138-e141. doi:10.1016/S1473-3099(20) 30052-9

8. Patel MK, Gacic-Dobo M, Strebel PM, et al. Progress toward regional measles elimination-worldwide, 2000-2015. MMWR Morb Mortal Wkly Rep. 2016;65(44):1228-1233. doi:10.15585/mmwr. mm6544a6

9. Paniz-Mondolfi AE, Tami A, Grillet ME, et al. Resurgence of vaccine-preventable diseases in Venezuela as a regional public health threat in the Americas. Emerg Infect Dis. 2019;25(4):625. doi:10.3201/eid2504.181305

10. Litvoc MN, Lopes MIBF. From the measles-free status to the current outbreak in Brasil. Rev Assoc Med Bras. 2019;65(10):1229-1230. doi:10.1590/1806-9282.65.10.1129 
11. Ochani RK, Yasmin F, Tariq A, Shaikh A. Philippines hit by deadly measles outbreak- a wakeup call? Infez Med. 2019;27(3):353-354.

12. Mc Donald HI, Tessier E, White JM, et al. Early impact of the coronavirus disease (COVID 19) pandemic and physical distancing measures on routine childhood vaccination in England, january to april 2020. Eurosurveillance. 2020;25(19):2000848.

13. WHO. Repository for supplementary immunization activities. Geneva: World Health Organization; 2020. Available from: www. who.it. Accessed November 25, 2020.

14. Thompson KM. Evolution and use of dynamic transmission models for measles and rubella risk and policy analysis. Risk Anal. 2016 doi:10.1111/risa.12637

15. WHO. Strategic Response Plan for the Measles Emergency in the WHO European Region. September 2019-December 2020. Worl Health Organization. Regional Office for Europe; 2019.

16. De G. In Fields Virology. Fields BN, Howley PM, Cohen JI, Knipe DM, eds. Wolters Kluver/Lippincott Williams \& Wilkins; 2013:1042-1069.

17. Perry R, Murray JS, Gacic-Dobo M, et al. Progress toward regional measles elimination - worldwide, 2000-2013. MMWR Morb Mortal Wkly Rep. 2014;63:1034-1038.

18. Mulders M, Rota PA, Icenogle JP, et al. Global measles and rubella laboratory network support for elimination goals, 2010-2015. MMW. 2016;65(17):438-442.

19. WHO. Global Vaccine Action Plan 2011-2020. 2013:1-149

20. Bozzola E, Spina G, Russo R, et al. Mandatory vaccinations in European countries, undocumented information, false news and the impact on vaccination uptake: the position of the Italian pediatric society. Ital J Pediatr. 2018;44(1):67. doi:10.1186/s13052-0180504-y

21. Phadke VK, Bednarczyk RA, Salmon DA, Omer SB. Association between vaccine refusal and vaccine-preventable diseases in the United States: a review of measles and pertussis. JAMA. 2016;315 (11):1149-1158. doi:10.1001/jama.2016.1353

22. Dhoyunk K. Mechanism of Maternal Antibody Inhibition and Vaccination Strategies in the Presence of Maternal Antibodies. Graduate Program in Molecular, Cellular and Developmental Biology. The Ohio State University; 2012.

23. Fredrickson DD, Davis TC, Arnould CL, et al. Childhood immunization refusal: provider and parent perceptions. Fam Med. 2004;36:431-439.

24. Gust DA, Kennedy A, Shui I, Smith PJ, Nowak G, Pickering LK. Parent attitudes toward immunizations and healthcare providers the role of information. Am J Prev Med. 2005;29(2):105-112. doi:10.1016/j.amepre.2005.04.010

25. Freed GL, Clark SJ, Butchart AT, Singer DC, Davis MM. Parental vaccine safety concerns in 2009. Pediatrics. 2010;125(4):654-659. doi:10.1542/peds.2009-1962

26. Seeber L, Michl B, Rundblad G, et al. A design thinking approach to effective vaccine safety communication. Curr Drug Saf. 2015;10 (1):31-40. doi:10.2174/157488631001150407105400

27. Goldacre B. Bad Science. Fourth Estate; 2008.

28. Madsen KM, Hviid A, Vestergaard M, et al. A population-based study of measles, mumps, and rubella vaccination and autism. $N$ Engl $J \quad$ Med. 2002;347(19):1477-1482. doi:10.1056/ NEJMoa021134

29. Jain A, Marshall J, Buikema A, Bancroft T, Kelly JP, Newschaffer CJ. Autism occurrence by MMR vaccine status among US children with older siblings with and without autism. JAMA. 2015;313(15):1534-1540. doi:10.1001/jama.2015.3077

30. COVER. Table 7: Completed Primary Courses: Percentage of Children Vaccinated by Their Second Birthday Against Measles Mumps and Rubella (MMR), England, 1988-89 to 2016-17. 2017.

31. Ford JA, Mahgoub H, Shankar AG. Vaccine acceptance: the UK perspective. Hum Vaccin Immunother. 2013;9(12):2658-2660. doi: $10.4161 / \mathrm{hv} .26411$
32. Olive JK, Hotez PJ, Damania A, Nolan MS. The state of the antivaccine movement in the United States: a focused examination of nonmedical exemptions in states and counties. PLoS Med. 2018;15 (6):e1002578. doi:10.1371/journal.pmed.1002578

33. Clemmons NS, Gastanaduy PA, Fiebelkorn AP, Redd SB, Wallace GS. Centers for disease control and prevention (CDC). Measles-United States, january 4-april 2, 2015. MMWR Morb Mortal Wkly Rep. 2015;64(14):373-376.

34. Cataldi JS, Dempsey AF, O'Leary ST. Measles, the media, and MMR: impact of the 2014-15 measles outbreak. Vaccine. 2016;34 (50):6375-6380. doi:10.1016/j.vaccine.2016.10.048

35. Friederichs V, Cameron JC, Robertson C. Impact of adverse publicity on MMR vaccine uptake: a population based analysis of vaccine uptake records for one million children, born 1987-2004. Arch Dis Child. 2006;91(6):465-468. doi:10.1136/adc.2005.085944

36. Dube E, Laberge C, Guay M, Bramadat P, Roy R, Bettinger J. Vaccine hesitancy: an overview. Hum Vaccin Immunother. 2013;9 (8):1763-1773. doi:10.4161/hv.24657

37. Dube E, Vivion M, MacDonald N. Vaccine hesitancy, vaccine refusal and the anti-vaccine movement: influence, impact and implications. Expert Rev Vaccines. 2015;14(1):99-117. doi:10.1586/14760584.2015.964212

38. Horne Z, Powell D, Hummel JE, Holyoak KJ. Countering antivaccination attitudes. Proc Natl Acad Sci $U$ S A. 2015;112 (33):10321-10324. doi:10.1073/pnas.1504019112

39. Gunartne K, Coomes EA, Haghbayan H. Temporal trends in anti-vaccine discourse on twitter. Vaccine. 2019;37(35):4867-4871. doi:10.1016/j.vaccine.2019.06.086

40. Schmidt AL, Zollo F, Scala A, Betsch C, Quattrociocchi W. Polarization of the vaccination debate on facebook. Vaccine. 2018;36(25):3606-3612. doi:10.1016/j.vaccine.2018.05.040

41. Zipprich J, Winter K, Hacker J, Xia D, Watt J, Harriman K. Measles outbreak- California, december 2014-february 2015. MMWR Morb Mortal Wkly Rep. 2015;64(6):153-154.

42. Cook J, Lewandowsky S. The Debunking Handbook. 2011. doi:10.1017/CBO9781107415324.004

43. Hample D, Hample JM. Persuasion about health risks: evidence, credibility, scientific flourishes, and risk perceptions. Argum Advocacy. 2014;51(1):17-29. doi:10.1080/00028533.2014.11821836

44. Gesser-Edelsburg A, Diamant A, Hijazi R, Mesch GS. Correcting misinformations by health organizations during measles outbreaks: a controlled experiment. PLoS One. 2018;13(12):e0209505. doi:10.1371/journal.pone.0209505

45. Slovic P, Finucane M, Peters E, MacGregor D. Risk as analysis and risk as feelings: some thoughts about affect, reason, risk and rationality. Risk Anal. 2004;24(2):311-322. doi:10.1111/j.02724332.2004.00433.x

46. Jarrett C, Wilson R, O'Leary M, Eckersberger E, Larson HJ. Strategies for addressing vaccine hesitancy-a systematic review. Vaccine. 2015;33(34):4180-4190. doi:10.1016/j.vaccine.2015.04.040

47. Zollo F. Dealing with digital misinformation: a polarised context of narratives and tribes. Efsa J. 2019;17(suppl 1):e170720. doi:10.2903/ j.efsa.2019.e170720

48. Sunstein CR. The law of group polarization. $J$ Polit Philos. 2002;10 (2):175-195. doi:10.1111/1467-9760.00148

49. Gesualdo F, Zamperini N, Tozzi AE. To talk better about vaccines, we should talk less about vaccines. Vaccine. 2018;36(34):5107-5108. doi:10.1016/j.vaccine.2018.07.025

50. Feemster KA. Building vaccine acceptance through communication advocacy. Hum Vaccin Immunother. 2020;16(5):1004-1006. doi:10.1080/21645515.2020.1746603

51. The LC. Vaccine hesitancy: a generation at risk. Lancet Child Adolesc Health. 2019;3(5):p281. doi:10.1016/S2352-4642(19) 30092-6

52. Glanz JM, Wagner NM, Narwaney KJ, et al. Web-based social media interventions to increase vaccine acceptance. Pediatrics. 2017;140 (6):e20171117. doi:10.1542/peds.2017-1117 
53. Broniatowski DA, Hilyard KM, Vaccine DM. Effective vaccine communication during the Disneyland measles outbreak. Vaccine. 2016;34(28):3225-3228. doi:10.1016/j.vaccine.2016.04.044

54. Gesualdo F, Marino F, Mantero J, et al. The use of web analytics combined with other data streams for tailoring online vaccine safety information at global level: the vaccine Safety Net's web analytics project. Vaccine. 2020;38(41):6418-6426. doi:10.1016/j.vaccine. 2020.07.070
55. ECDC. Conducting health communication activities on MMR vaccination; 2010. Available from: www.ecdc.europa.eu. Accessed November 25, 2020.

56. Larson HJ, de Figueiredo A, Xiahong Z, et al. The state of vaccine confidence 2016: global insights trough a 67-country survey. EBioMedicine. 2016;12:295-301.

\section{Publish your work in this journal}

Risk Management and Healthcare Policy is an international, peerreviewed, open access journal focusing on all aspects of public health, policy, and preventative measures to promote good health and improve morbidity and mortality in the population. The journal welcomes submitted papers covering original research, basic science, clinical \& epidemiological studies, reviews and evaluations, guidelines, expert opinion and commentary, case reports and extended reports. The manuscript management system is completely online and includes a very quick and fair peer-review system, which is all easy to use. Visit http://www.dovepress.com/testimonials.php to read real quotes from published authors. 